\title{
What are the Major Transitions?
}

\author{
Matthew Herron ${ }^{1, *}$ \\ 1 School of Biological Sciences, Georgia Institute of Technology, Atlanta, \\ GA 30332 \\ *xprinceps@gmail.com
}

\begin{abstract}
The 'Major Transitions in Evolution' (MTE) framework has emerged as the dominant paradigm for understanding the origins of life's hierarchical organization, but it has been criticized on the grounds that it lacks theoretical unity, that is, that the events that make up the category do not constitute a natural kind. I agree with this criticism, and I argue that the best response is to modify the category so that it does approximate a natural kind. Specifically, I recommend defining major transitions as all those, and only those, events and processes that result in the emergence of a new level of individuality. Two sorts of changes will be required to achieve this. First, events and processes that do not meet this criterion, such as the origins of the genetic code and of human language, should be excluded. Second, events and processes that do meet the criterion, but which have generally been neglected, should be included. These changes would have the dual benefits of making MTEs a philosophically coherent category and of increasing the sample size on which we may infer trends and general principles that may apply to all MTEs.
\end{abstract}

\section{Introduction}

I grew up thinking that Pluto was a planet. In 2006, however, the International Astronomical Union formally defined the word "planet" 1], and because it had failed to "clear the neighborhood" around its orbit, Pluto didn't make the cut. 2 . As a result, textbooks will have to be revised, posters redesigned, museum displays rebuilt...there are real economic costs to this change. Why, then, did they do it? Pluto has changed little since its discovery in 1930. Why couldn't the IAU leave well enough alone?

Humans classify the things we observe. We all agree that celestial bodies, biological organisms, and musical compositions should be sorted into categories, taxa, or genres, but why? One reason is that we think there is value in grouping like things, because doing so allows us to make generalizations. Mammals are warm-blooded and produce milk. Rockabilly combines elements of blues and country music. Planets are roundish, orbit the sun, and clear the neighborhood around their orbit. 1] Generalizing, in turn, allows us to ask questions about the group as a whole, with some hope that the answers will apply to all of its members. Why are mammals warm-blooded? Who were the pioneers of rockabilly? How do planets form?

As we discover more things, or more properties of known things, sometimes we have to reconsider the boundaries of a category. Classifications that we thought were discrete and unambiguous turn out to grade into each other and to include marginal cases, requiring refinement or revision of the criteria for inclusion. The discovery of 
monotremes, for example, required removing live birth from the criteria for mammals. The discovery of over 1000 Trans-Neptunian Objects required refining the criteria for planets. [1] Genre-straddling musicians spark debates over, for example, whether Lil Nas X's "Old Town Road" should be ranked on country music charts. 3 .

The reason, in a nutshell, that the IAU couldn't leave well enough alone is that the existing classification would have identified all of the trans-Neptunian Objects as planets, and there was no way short of blatant gerrymandering to change the definition to exclude them but include Pluto. So why not just include all of the Trans-Neptunian Objects? Celestial bodies that fail to clear their orbital neighborhoods differ from planets in both their mechanisms of formation and their roles in the orbital mechanics of the solar system. Classifying bodies as planets is useful because it allows us to generalize about these mechanisms and roles and to have some confidence that our generalizations will be true for all planets, including extrasolar planets. Including trans-Neptunian Objects that fail to clear their orbital neighborhoods would invalidate some of these generalizations, making the classification less useful.

One of the classifications that has proved useful in biology is that of the so-called 'Major Transitions in Evolution' (MTE). The MTE framework is an attempt to explain the hierarchical structure of life on Earth: genes within chromosomes, chromosomes within cells, cells within cells (eukaryotic cells), individuals within sexual partnerships, cells within multicellular organisms, and organisms within societies.

The best-known effort to unify the origins of these relationships is a book by John Maynard Smith and Eörs Szathmáry, The Major Transitions in Evolution. [4 First published in 1995, the book focused on the origins of these hierarchical levels, uniting them into a category based on the shared criteria that they change "the way in which genetic information is transmitted between generations," namely that "... entities that were capable of independent replication before the transition can replicate only as part of a larger whole after it." For example, after a transition from unicellular to multicellular organisms (there have been several), cellular reproduction either contributes to the growth of the organism or to production of new multicellular organisms.

Rick Michod has revised this idea, focusing less on reproduction and more on units of fitness. 5 7 An edited volume intended to update the Major Transitions framework, including chapters by Szathmáry 8 and Michod 9 was published in 2011. More recently, Szathmáry 10 himself has updated the framework he developed with Maynard Smith, removing sex from the list, adding endosymbiotic events, and folding in David Queller's fraternal/egalitarian distinction (which, incidentally, first appeared in a review of The Major Transitions in Evolution). 11

Thus, the MTE framework has itself evolved, diversifying into several different versions that nevertheless retain some ancestral characters. I will briefly review these variants, focusing on the plesiomorphies that unite them and the apomorphies that distinguish them. I will argue that the most useful definition of an MTE is 'an evolutionary change that gives rise to a new level of individuality,' and I will discuss the ambiguities that follow from this definition. I will explore the practical implications of applying this definition and explore ambiguous cases near the margins. Finally, I will summarize what we have learned, and what we still hope to learn, about the shared and unique features of the Major Transitions.

\section{The radiation of frameworks}

Maynard Smith and Szathmáry's Major Transitions framework was foreshadowed by a long tradition of viewing life as hierarchically organized and, in some cases, of viewing more inclusive units as having evolved from less inclusive units. August Weismann, for example, recognized that multicellular organisms had evolved from unicellular [12, 
and further postulated that cells were made up of groups of molecules that he called biophors. [13] Similarly, Herbert Spencer argued that cells must consist of subcellular components he called physiological units, which were formed by "further compounding of highly compound molecules." [14 p. 226] William Morton Wheeler extended the hierarchy to include societies of social insects and of humans, both of which he considered real organisms. 15] John Tyler Bonner wrote of "leaps from one level of complexity to the next," including the origins of life, of eukaryotes, of multicellularity, and of sociality (Table 1). 16 Leo Buss interpreted the hierarchy of life as one of increasingly inclusive units of selection, "species composed of populations, populations of individuals, individuals of cells, cells of organelles, organelles of genomes, genomes of chromosomes, and chromosomes of genes," and he proposed an evolutionary mechanism for its origin (Table 1). 17, pp. 183-184]

The first comprehensive effort to understand these transitions within a unified framework, though, was Maynard Smith and Szathmáry's 1995 book The Major Transitions in Evolution (Table 1). 44 This book, and an accompanying paper in Nature [18, attempted to explain the existence of biological units with different levels of complexity as the result of a series of events in which existing units became integrated into new, higher-level units. The most important feature of these events was that "entities that were capable of independent replication before the transition can replicate only as part of a larger whole after it." [4. p. 4] Other important features included division of labor among the lower-level units and changes in the way information is transmitted.

In his review of The Major Transitions in Evolution, David Queller observed that Maynard Smith and Szathmáry's framework included two distinct sorts of transitions, with different initial advantages and eventual outcomes. [11 Fraternal transitions occur among genetically similar units, such as the cells in a multicellular organism or the ants in a colony, and a reproductive division of labor can evolve through kin selection. Egalitarian transitions involve unlike or unrelated units, such as the Archaean and bacterium that combined to form the eukaryotic cell, and although both partners benefit from the alliance, both retain the ability to reproduce.

Richard Michod has focused on the subset of the major transitions that, in his view, result in a new unit of selection, including those "from individual genes to networks of genes, from gene networks to bacteria-like cells, from bacteria-like cells to eukaryotic cells with organelles, from cells to multicellular organisms, and from solitary organisms to societies" (Table 1). [6, p. 7] His more recent works also include the origins of sexual reproduction. [9] Michod's focus on units of selection is consistent with the views of Buss [17, pp. 183-184] but narrower than that of Maynard Smith and Szathmáry, who acknowledged that some, but not all, of their major transitions involved a change in the units of evolution; for example, the origin of the genetic code does not fit this scheme [4, p. 12]. In accordance with this narrower focus, Michod prefers to call the transitions in his framework evolutionary transitions in individuality (ETIs) rather than major transitions. $5,7,9$

In 2011, Brett Calcott and Kim Sterelny published an edited volume, The Major Transitions in Evolution Revisited, with a diverse set of perspectives. [19] Several of the authors accepted Michod's view either explicitly or implicitly (by only considering the subset of the major transitions that are also transitions in individuality). $9,20,21$ Others advocated expanding the framework to include, for example, the endosymbiotic origins of plastids 8 22, the Cambrian explosion [23], the mutualistic association between corals and zooxanthellae 22, and other evolutionary innovations within the metazoa 8].

Twenty years after the publication of The Major Transitions in Evolution, Szathmáry revisited the topic, presenting his conception for a "Major evolutionary transitions theory 2.0" (Table 11) [10] He advocated two major changes: the removal of sex and the inclusion of plastid acquisition. Sex, he argued, was best viewed not as a separate 
major transition, but "as a coevolving form of maintenance or transformation of an emerging higher-level evolutionary unit," namely the eukaryotic cell. [10, p. 10108] Since plastids are now understood to result from endosymbiotic events analogous to the acquisition of the mitochondrion, Szathmáry argued for the inclusion of both primary and recursive (secondary, tertiary, and quaternary) plastid acquisition events within the major transitions framework.

Table 1. A partial list of versions of the major transitions or transitions in individuality framework. This is not intended to be a comprehensive list, but a sampling of works that have attempted to present or revise a framework for the evolution of life's hierarchical structure. $4,4,9,10,16,17,24,25$.

\begin{tabular}{|c|c|c|c|c|c|c|c|c|}
\hline Origin of... & $\begin{array}{l}\text { Bonner } \\
1974\end{array}$ & $\begin{array}{l}\text { Buss } \\
1987\end{array}$ & $\begin{array}{l}\text { Maynard } \\
\text { Smith \& } \\
\text { Szathmáry } \\
1995\end{array}$ & $\begin{array}{l}\text { Michod } \\
2011\end{array}$ & $\begin{array}{l}\text { Bourke } \\
2011\end{array}$ & $\begin{array}{l}\text { Szathmáry } \\
2015\end{array}$ & $\begin{array}{l}\text { West } \\
\text { et al. } \\
2015\end{array}$ & This work \\
\hline Eukaryotes & $\mathrm{X}$ & $\mathrm{X}$ & $\mathrm{X}$ & $\mathrm{X}$ & $\mathrm{X}$ & $\mathrm{X}$ & $\mathrm{X}$ & $\mathrm{X}$ \\
\hline Multicellularity & $\mathrm{X}$ & $\mathrm{X}$ & $\mathrm{X}$ & $\mathrm{X}$ & $\mathrm{X}$ & $\mathrm{X}$ & $\mathrm{X}$ & $\mathrm{X}$ \\
\hline Eusociality & $\mathrm{X}$ & $\mathrm{X}$ & $\mathrm{X}$ & $\mathrm{X}$ & $\mathrm{X}$ & $\mathrm{X}$ & $\mathrm{X}$ & $\mathrm{X}$ \\
\hline Protocells/life & $\mathrm{X}$ & $\mathrm{X}$ & $\mathrm{X}$ & $\mathrm{X}$ & $\mathrm{X}$ & $\mathrm{X}$ & & $\mathrm{X}$ \\
\hline Chromosomes & & $\mathrm{X}$ & $\mathrm{X}$ & $\mathrm{X}$ & & $\mathrm{X}$ & $\mathrm{X}$ & $\mathrm{X}$ \\
\hline Sex & & $\mathrm{X}$ & $\mathrm{X}$ & $\mathrm{X}$ & $\mathrm{X}$ & & & \\
\hline Language/memes & & $\mathrm{X}$ & $\mathrm{X}$ & & & $\mathrm{X}$ & & \\
\hline Genetic code & & & $\mathrm{X}$ & & & $\mathrm{X}$ & & \\
\hline Plastids & & & & & & $\mathrm{X}$ & $\mathrm{X}$ & $\mathrm{X}$ \\
\hline Mutualisms & & & & & $\mathrm{X}$ & & $\mathrm{X}$ & $\mathrm{X}$ \\
\hline Colonial animals & & $\mathrm{X}$ & & & $\mathrm{X}$ & & & $\mathrm{X}$ \\
\hline
\end{tabular}

\section{Natural kinds}

There is something philosophically muddled and scientifically casual about [the major transitions framework]...The fault has been the yielding to theoretical inconsistency. Enough is enough. [26, p. 32]

From the beginning, the major transitions framework has been criticized for lumping together dissimilar events. Maynard Smith and Szathmáry acknowledged that their list was somewhat arbitrary [4, and Queller, in his review of The Major Transitions, suggested that it was really two books, one describing changes in the mechanisms of inheritance and one addressing the evolution of cooperation. [11] In their chapter in The Major Transitions in Evolution Revisited, Daniel McShea and Carl Simpson argued that Maynard Smith and Szathmáry's list of major transitions lacks theoretical unity and needs to be revised. 26 Michod, in the same volume, defended his shorter list of transitions in individuality on the grounds that, unlike Maynard Smith and Szathmáry's list, his constituted a natural kind (a category whose members share fundamental similarities). 9 More recently, O'Malley and Powell pointed out that both the original and revised forms of the major transitions framework fail as natural kinds, shoehorning in events that fail to meet any common set of criteria and failing to include some events that do. 27

I agree with McShea and Simpson: enough is enough. To be most useful, the MTE framework should be modified so that the events and processes included approximate a natural kind. As the IAU did with planets, we should define the qualifications, then include or exclude particular examples based on the degree to which those qualifications are met. The criterion that comes closest to current and historical usage is 'an 
evolutionary change that gives rise to a new level of individuality.' This is consistent with Buss's emergence of new levels of selection [17], since most evolutionary concepts of individuality revolve around units of selection or of adaptation. $11,20,28,33$ It does not apply to all of Maynard Smith and Szathmáry's transitions (as they acknowledge), but it does apply to those that meet the criterion they considered most important, the shift from independent to group replication [4] and is consistent with Maynard Smith's earlier work on the topic. 34 It is also the criterion explicitly advocated by Michod and colleagues [5,7,9] and by West et al. 25]. Even Szathmáry seems to have recently come around to viewing major transitions as transitions in individuality. 35]

\section{Applying the definition}

Each polypus, though closely united to its brethren, has a distinct mouth, body, and tentacula. Of these polypi, in a large specimen, there must be many thousands; yet we see that they act by one movement; that they have one central axis connected with a system of obscure circulation; and that the ova are produced in an organ distinct from the separate individuals. Well may one be allowed to ask, what is an individual? [36, p. 117], referring to a colonial cnidarian sea pen.

Having chosen a criterion, we should apply it consistently. Two kinds of changes will be needed. First, events and processes that don't fit the criterion of resulting in a new level of individuality should be ruthlessly pruned. The most obvious candidate for expulsion is the origin of the genetic code. The fact that this transition is rarely discussed in the MTE framework constitutes an implicit acknowledgement of its poor fit, and various authors have pointed out explicitly that it does not belong. 9, 25, 27] Similarly, the origin of language has been largely absent from discussions of major transitions, and several authors have argued that it should be excluded for the sake of theoretical consistency. 9, 25, 27. Neither innovation meets what Maynard Smith and Szathmáry themselves identify as their most important criterion, the shift from independent to group replication. 4] Two of these things are not like the others; let us follow the IAU's example and excise that which does not belong.

Surprisingly, several recent proposals have gone the opposite direction. Various modifications have been proposed that would, if implemented, exacerbate the lack of theoretical unity by adding evolutionary changes that have very little in common with the transitions traditionally included within the MTE framework. Proposed additions include the origin of oxygenic photosynthesis and the resulting oxygenation of the Earth's atmosphere [8,27]; the origin of a nervous system in animals [37]; the Cambrian Explosion [23]; the origin of closed circulation systems in vertebrates, annelids, and cephalopods 8]; the origin of a rigid cell wall [8]; the origin of an exoskeleton in Ecdysozoa [8]; and the origin of the immune system. 8]

All of these proposed additions are undoubtedly important evolutionary events, but none of them belong in the MTE framework. They don't meet Buss's criteria of transitions between units of selection [17, p. viii], Maynard Smith and Szathmáry's of independently replicating entities shifting to group replication [4], or Michod's of the emergence of new evolutionary individuals. [5 7, 9] Adding the origins photosynthesis, nervous systems, cell walls, etc. to the MTE framework is analogous to expanding the definition of planets to include not only trans-Neptunian objects but comets, asteroids, and moons as well.

The justification for most of the proposed additions is their outsized effects on the biosphere and on subsequent evolutionary processes. Importance, though, is a lousy criterion for grouping evolutionary processes. The MTE framework is a part 
of evolutionary theory in the same way that behavioral ecology, quantitative genetics, evo-devo, and inclusive fitness are parts of evolutionary theory. Each seeks to explain some aspect of biology and includes within its purview all of the relevant examples without regard for the magnitude of their effect. Behavioral ecology does not exclude behaviors unique to a single species, nor does evo-devo exclude monotreme development because monotremes have not experienced a large adaptive radiation. In each case, the fit of a particular process to a subfield is a matter of similarity to other processes in the same subfield, not of perceived importance.

Importance is a lousy criterion because it is based on outcomes, not on fundamental similarities. Maynard Smith and Szathmáry's list was not intended to be a list of the most important evolutionary changes (in spite of the title of their book). In fact, they specifically exclude consideration of such "major phenotypic changes" as terrestrialization and the origins of vision, flight, and homeothermy. [4] The framework that explains processes as diverse as the evolution of multicellularity, of nervous systems, and of cell walls might be best described as 'evolutionary biology'; no smaller category will suffice. Aside from preventing theoretical unity, using importance as a criterion precludes consideration of recent or in-progress transitions, the eventual importance of which we can't yet evaluate.

The second kind of change that will be needed to make the MTE framework approximate a natural kind is to include events and processes that meet the criteria of resulting in a new level of individuality, but which have generally been ignored. Some such additions have already been suggested. For example, the endosymbiosis of a cyanobacterium that resulted in the primary acquisition of a chloroplast, and secondary and higher-order acquisitions of eukaryotic algae, are egalitarian transitions that parallel the endosymbiotic origin of mitochondria. Although they have been relatively neglected in the MTE literature, several authors have pointed out that they belong. $10,24,27,38,39$ The relatively recent domestication of a cyanobacterium by the filose amoeba Paulinella, which may represent a second origin of a primary chloroplast, has also been nominated for inclusion. 10,40

Other kinds of multispecies coalitions have also been proposed for inclusion in the MTE framework. Benjamin Kerr and Joshua Nahum, for example, argue that the association between corals and single-celled algae called zooxanthellae qualifies. 22 Andrew Bourke and Szathmáry both consider the origins of lichens as major transitions 10,24 , and Szathmáry also includes the Buchnera-aphid symbiosis within his 'Major Transitions 2.0'. 10]

Symbiotic associations occupy a spectrum of degrees of intimacy from casual to mitochondria. Which of these qualify as a composite individual, or holobiont, will undoubtedly differ among readers. Most would, I think, agree that free-living protists, with their bacterial and archaeal components, qualify; few would, I think, extend that to oxpeckers and wildebeest. In between, there is a range of associations that may or may not constitute major transitions. What I am advocating is a standard by which we can judge: does the association constitute a composite individual? If so, it should be included within the MTE framework.

Among the fraternal transitions, the most egregious oversight is the exclusion of colonial animals, such as marine invertebrates within the zoantharian and anthozoan corals, hydrozoans, bryozoans, graptolites, and ascidians. Many have differentiated zooids, some even with reproductive specialization, a striking parallel to differentiated cells in multicellular organisms. Buss hints that he does consider such 'metameric organisms' to constitute a new level of selection 17, p. 195], and Bourke argues explicitly for their inclusion. 24 As with the putative egalitarian transitions discussed above, the question of whether or not a coral or graptolite colony is the outcome of a major transition hinges on whether or not it qualifies as an individual. The most integrated of 


\section{Ambiguities}

We designate something as an organism, not because it is $n$ steps up on the ladder of life, but because it is a consolidated unit of design, the focal point where lines of adaptation converge. It is where history has conspired to make between-unit selection efficacious and within-unit selection impotent. 11, p. $187]$

I do not imagine or intend that this essay will end disagreements about what processes belong in the MTE framework. I have advocated for an explicit criterion - those that result in a new level of individuality - but that, of course, raises a new problem: what constitutes a new level of individuality? This in turn raises the question of what is an individual, about which too much has been written to review here.

Since the MTE framework, in any version, is a set of questions about evolutionary processes, an evolutionary concept of individuality is the most relevant. The concept of individuality that has been embraced by most MTE authors is that of units of selection. 4142 Buss [17, Maynard Smith and Szathmáry [4], Michod 5], Szathmáry 10], and West and colleagues 25, among many others, have all employed some form of this criterion.

Even if we agree that a major transition is an evolutionary process that results in a new unit of selection, we will still be left with marginal cases. Individuality comes in degrees, and any line we draw will be arbitrary 20, 43, 44. In fact, since most such transitions probably play out over geological time scales, while we can generally only see a snapshot, we should expect to see many incomplete transitions at any given time. These may be on their way to a complete transition, or they may be stable arrangements in their own right. 44]

Intermediate cases, in which a new level of individuality has partially or debatably emerged, should be part of the MTE conversation. They are our best window into the processes and intermediate states that occur during a major transition. When we look at only the ends of a spectrum, we may miss the middle, which is where the interesting stuff is happening.

\section{Let's talk about sex}

[S]ex requires the coordination of two individuals, and, so implies the existence of a higher-level unit, the reproductive pair...Though I argue that viewing sex as an ETI may help integrate different perspectives on the problem of the evolution of sex, there are several senses in which the evolution of sex is not an ETI in the same sense as, say, multicellularity. 9, p. 186]

I have so far largely ignored one of Maynard Smith and Szathmáry's [4] transitions, that from asexual to sexual populations. Whether or not the origin of sex should be included within the MTE framework is a more difficult question than those I considered in the previous sections. The origins of the genetic code and of language are clearly not origins of new levels of individuality. The origins of plastids and other endosymbionts, of lichens, and of colonial marine invertebrates clearly are.

The evolution of eukaryotic sex was one of Maynard Smith and Szathmáry's major transitions. 44 Michod generally includes it within his evolutionary transitions in individuality, though he admits that there are important differences between sex and other such transitions. [9 Bourke also includes sex as a transition in individuality. 24 On the other 
hand, Queller pointed out the poor fit of sex within the major transitions framework [11, Szathmáry demoted it to an subprocess within the evolution of eukaryotes 10], and West and colleagues excluded it altogether. 25

The evolution of sex has some fundamental similarities to that of eukaryotes, multicellularity, and eusociality, but important differences from these processes as well. But viewing major transitions in the way I advocate gives us a grip on the question. Whether or not sex belongs in the major transitions framework should be decided on the same basis as other transitions: does it result in a new unit of selection?

My assessment is that in most cases, it does not. In the view I have outlined here, sex would be a major transition if the mated pair constitutes a unit of selection. Indeed, these are the grounds on which Michod [9, p. 186] justifies viewing sex as an ETI: "...fitness is a property of the reproductive pair, not of individual organisms. In this sense, the reproductive pair is the real evolutionary individual in obligate sexual species."

It is true that the members of a mated pair often cooperate. But is the mated pair an evolutionary individual in the sense of a unit of selection? Each has its own genetic interest, namely to increase the representation of the alleles it carries in future generations. These different interests cause conflicts, both intra- and interlocus, and in some cases these conflicts are so severe as to be fatal. 45. Often each partner has its own preference regarding how often and with whom the other mates, and arms races occur in an escalating contest to enforce (or escape enforcement of) these preferences. 45 Even in cases of strict genetic monogamy, each partner has a different optimal level of resource investment, which manifests as conflicts over such things as clutch size and parental care.

Perhaps the strongest argument against viewing the mated pair as a unit of selection is that the members' fitnesses are not generally the same. Although their fecundities are certainly linked, they are only likely to be equal in cases of strict genetic monogamy. Worse, their viabilities are, in most cases, quite independent, since the death of one does not imply the death of the other.

The best example supporting the view of sex as an MTE is probably cases of so-called "sexual parasites" as found in some anglerfish. [46 In such cases, the male may be a half million times smaller than the female, with whom he permanently fuses, eventually devolving to little more than a gonad. Even so, the male and female retain separate reproductive interests, as the female often multiply mates 47, while the male would surely prefer an exclusive partnership in which he fertilizes all of the female's eggs. Thus, even in the best case scenario, the mated pair is a poor candidate for individuality, undermining the case for including sex in the MTE framework.

\section{The payoff: common themes}

There is sufficient formal similarity between the various transitions to hold out the hope that progress in understanding any one of them will help illuminate the others. [18, p. 23]

Rocky planets are generally thought to form inside, and gas giants outside, the snow line (the distance from the central protostar at which water condensation becomes possible). 48 Trans-Neptunian objects (including Pluto) do not fit this generalization, because they formed through fundamentally different processes. [49] If current models of planetary formation are correct, we can expect to find exoplanets dominated by rocky planets inside, and gas giants outside, their systems' snow lines. A contrary finding would imply that planetary migration is common, that snow lines frequently move, or that current models are wrong. [50] By choosing objective criteria and applying 
them consistently, the IAU has made planets something like a natural kind, allowing meaningful generalizations and increasing the utility of the category.

Similarly, if we define MTEs as something like a natural kind, we can hope to fulfill Szathmáry and Maynard Smith's [18] vision by finding principles that apply across levels of organization, from chromosomes to societies. Some of these principles have already been inferred, for example that cooperation among the lower-level units plays an important role in the emergence of a higher-level individual and that such transitions often lead to division of labor among the lower-level units. [4] If these principles truly are general, we can expect that they will hold wherever life exists. A contrary finding would force a revision of our models of major transitions, or at least a narrowing of their scope.

Defining MTEs this way allows us to ask questions about the processes and outcomes of major transitions that are not possible if those processes and outcomes are treated as criteria. Is cooperation among the lower-level units always necessary? Under what conditions do major transitions lead to division of labor? How consistently, and in what sense, do major transitions involve a change in the mechanism of inheritance?

By excluding criteria based on importance, we can also ask meaningful questions about the outcomes of major transitions. What are the factors that determine whether or not a major transition leads to a large adaptive radiation, or to biosphere-altering ecological effects? These questions are rendered meaningless if large adaptive radiations and biosphere-altering effects are qualifications for major transitions, just as using 'has a biosphere' as a criterion for a celestial body to qualify as a planet would render meaningless questions about what kinds of planets can support life. We can only answer such questions by comparing transitions that have had important consequences with those that have not.

Including 'minor' major transitions (to borrow Grosberg and Strathmann's [51] terminology) also has the benefit of increasing sample size. In our search for trends and general principles, we now have access to a census (at least of known cases) instead of a biased sample. By examining similar processes, regardless of their outcomes, we can potentially learn about watershed transitions whose origins have been obscured by the eons, for example about the primary origin of chloroplasts from subsequent endosymbioses between heterotrophs and photoautotrophs. 52,53 What might we learn about intracellular symbioses by considering not only the origin of eukaryotes but of primary and higher-order plastids, insect endosymbionts (and endo-endosymbionts [54]), and algal cells that harbor Rickettsial bacteria [55]? What might we learn about clonal multicellularity from the spondylomoracean green alga Pyrobotrys [56], colonial choanoflagellates [57, and multicellular ciliates such as Zoothamnium, or about societies from bryophytes, graptolites, and corals?

\section{Objections}

The question can justifiably be raised whether we have a theory or not. I think we do, but with qualifications. [10, p. 10110]

Though they won't match the outcry over Pluto's exclusion, I expect that objections will be raised against the revision I have proposed. In this section, I will attempt to answer some that have already been raised and some that seem obvious.

Fraternal and egalitarian transitions [11] are fundamentally different processes. This is true, but they also have important characteristics in common, and historically both have been included in every version of the MTE framework (Table 1 ). Fraternal and egalitarian are useful subcategories within the larger category of major transitions, just as rocky and gas giant are useful subcategories within the larger category 
of planets. Both contribute to our understanding of the origins of life's hierarchical organization.

Viewing MTEs as transitions in individuality still lumps unique, one-off transitions with repeated, somewhat predictable events. 27. This too is true: some major transitions, such as the origins of multicellularity, have happened many times, while others, such as the endosymbiotic origin of eukaryotes, are unique. I don't see this as a problem. First of all, we don't know which events are, as O'Malley and Powell put it, "non-replicable, one-off events." 27, p. 163] The origin of eukaryotes is an obvious candidate, but we don't actually know that, if it hadn't happened in the Lokiarchaeota, something like eukaryotes would not have arisen elsewhere in the tree of life. Even if some of the transitions truly are non-replicable, though, this does not threaten the coherence of the category. Evolution, after all, is a combination of repeatable and contingent events.

Limiting MTEs to transitions in individuality excludes many events of fundamental importance to evolutionary processes and to the biosphere. 27, 58 Furthermore, including all of the transitions that result in a new level of individuality would mean including some events that have not (yet) had major evolutionary consequences. 27] These are only disadvantages if we think important consequences should be one of the criteria. I have explained in the previous sections why I think importance is better treated as an outcome than as a criterion. Having major consequences is surely contingent, and we can't say which recent transitions in individuality might have major consequences in the future. Furthermore, some events without major consequences have traditionally been included within the MTE framework, for example origins of multicellularity that have not led to major adaptive radiations or biosphere-altering ecological impacts, such as the volvocine algae.

Even in its revised form, the MTE framework remains progressivist, encouraging a 'monads to man' view of evolution. 27, 58] If this is true, it is an argument about how we should interpret the MTE framework, not about how we should define it. There is nothing inherently progressivist in recognizing that in some lineages, at some times, complexity, in the sense of the number of hierarchically nested levels [?,59], has increased. This is simply true. As Maynard Smith and Száthmary put it, "...there is surely some sense in which elephants and oak trees are more complex than bacteria, and bacteria than the first replicating molecules." [4. p. 23] A claim that such increases are universal or inevitable would be progressivist, but I am unfamiliar with any such claim in the peer-reviewed literature. Furthermore, in the view I advocate, humans occupy no privileged position, and the vast majority of MTEs - most endosymbiotic events, most origins of multicellularity, all origins of eusociality - did not lead to humans. If there is a 'highest' level (again, in terms of number of nested levels within), it would be that of quaking aspen groves, of eusocial insects and colonial marine invertebrates, of quaternary endosymbiosis-derived dinoflagellates, or possibly of ant 'supercolonies'. [?]

\section{Conclusions}

At the heart of my arguments is the simple observation that the history of life is a history of the elaboration of new self-replicating entities by the self-replicating entities contained within them (or the incorporation of some self-replicating entities by others). [17, p. vii]

I take the purpose of the major transitions framework to be explaining the hierarchical organization of life on Earth: societies made up of organisms, which are in turn made up of cells, cells within cells, and so on. 6, $6,17,21,24,60$ Each level of the hierarchy, from chromosomes to superorganisms, has or is thought to have emerged from components 
that were previously evolutionary individuals in their own right. I advocate including within the major transitions framework all those, and only those, events and processes in which a new level of individuality has emerged. Applying this criterion consistently would make the major transitions framework philosophically coherent, something like a natural kind. By including events and processes that have mostly been neglected, it would also increase the number of events from which we can draw inferences.

We do need a theoretical framework to explain other events of momentous consequence, such as the origin of the genetic code, the Cambrian explosion, the biotic oxygenation of Earth's atmosphere, and the origins of powered flight. Thankfully, such a framework exists. We could call it macroevolution, or simply evolutionary biology. No smaller category can hope to both explain such a wide variety of events and retain a semblance of coherence.

The course I advocate is a minimal intervention and much less invasive than other proposed treatments. Biologists and philosophers who write about the MTE framework, particularly those who seek general principles, have, by and large, already converged on something like the definition I advocate. In some cases this has been explicit; in others the authors have simply ignored MTEs that do not result in new levels of individuality. 20, 21, 24, 25, 35, 59,60 In fact, aside from their inclusion by Maynard Smith and Szathmary [4], the origins of the genetic code and language have played virtually no role in the development of the MTE framework, probably because there is nothing to be gained by their inclusion. The impracticality of including fundamentally different processes has, in other words, already driven the field toward adopting a coherent category. Recent proposals to include additional unrelated processes would reverse this trend, radically redefining the MTE framework into something unrecognizable and less useful. 24, 61].

There is no equivalent of the IAU for evolutionary biology, no final authority to impose a definition on the field. Definitions are not right or wrong anyway, but more or less useful. I have argued for a particular definition of major transitions on the grounds that it closely approximates a natural kind, making it more useful than less coherent definitions. Having a clear criterion will allow us to objectively evaluate whether a particular process or event constitutes a major transition, and grouping like processes and events will allow us to identify trends and general principles that may be true for all.

\section{Acknowledgments}

This work was inspired by a conversation with Maureen O'Malley and Russell Powell that played out on my personal blog, and I am grateful for their thoughtful discussion. Will Ratcliff, Peter Conlin, and Charles Lindsey provided useful feedback on an early draft. Some of the ideas herein undoubtedly originated in conversations with my Ph.D. advisor, Rick Michod, whose influence on my thinking has been profound. This material is based upon work while serving at the National Science Foundation and while supported by grants from NSF (DEB-1723293) and NASA (NNA17BB05A).

\section{References}

1. International Astronomical Union. IAU Resolution 6a: definition of Pluto-class objects. Technical report, 2006.

2. International Astronomical Union. IAU Resolution 5a: definition of a "planet" in the solar system. Technical report, 2006. 
3. E Leight. Lil Nas X's 'Old Town Road' was a country hit. Then country changed its mind. Rolling Stone, March 2019.

4. J Maynard Smith and E Szathmáry. The Major Transitions in Evolution. Oxford University Press, Oxford, 1995.

5. R E Michod and D Roze. Transitions in individuality. Proceedings: Biological Sciences, 264(1383):853-857, October 1997.

6. R E Michod. Darwinian Dynamics: Evolutionary Transitions in Fitness and Individuality. Princeton University Press, Princeton, NJ, 1999.

7. R E Michod. On the transfer of fitness from the cell to the multicellular organism. Biology \& Philosophy, 20(5):967-987, March 2005.

8. E Szathmáry and C Fernando. Concluding remarks. In B Calcott and K. Sterelny, editors, The Major Transitions in Evolution Revisited, pages 301-310. The MIT Press, Cambridge, MA, 2011.

9. R E Michod. Evolutionary transitions in individuality: multicellularity and sex. In The Major Transitions in Evolution Revisited, pages 169-197. MIT Press, 2011.

10. Eörs Szathmáry. Toward major evolutionary transitions theory 2.0. Proceedings of the National Academy of Sciences, pages 10104-10111, 2015.

11. David C Queller. Cooperators since life began. Quarterly Review of Biology, 72(2):184-188, 1997.

12. A Weismann. Essays Upon Heredity and Kindred Biological Problems (Authorised Translation). Clarendon Press, Oxford, 1889.

13. A Weismann. The Germ-Plasm: a Theory of Heredity (English Translation by $W$. N. Parker \& H. Rönnfeldt). Charles Scribner's Sons, New York, 1893.

14. H Spencer. The Principles of Biology, Volume 1. D. Appleton and Company, New York and London, 1910.

15. W M Wheeler. The ant-colony as an organism. Journal of Morphology, 22:307-325, 1911.

16. John Tyler Bonner. On Development: The Biology of Form. Harvard University Press, Boston, 1974.

17. Leo W Buss. The Evolution of Individuality. Princeton University Press, Princeton, 1987.

18. E Szathmáry and J Maynard Smith. The major evolutionary transitions. Nature, 374:227-232, 1995.

19. Brett Calcott and K. Sterelny. The Major Transitions in Evolution Revisited. Cambridge University Press, Cambridge, 2011.

20. Peter Godfrey-Smith. Darwinian populations and transitions in individuality. In Brett Calcott and K. Sterelny, editors, The Major Transitions in Evolution Revisited, pages 65-81. MIT Press, 2011.

21. C. Simpson. How many levels are there? How insights from evolutionary transitions in individuality help measure the hierarchical complexity of life. In B Calcott and K Sterelny, editors, The Major Transitions in Evolution Revisited, pages 199-225. The MIT Press, Cambridge, 2011. 
22. B Kerr and J Nahum. The evolution of restraint in structured populations: setting the stage for an egalitarian major transition. In B Calcott and K. Sterelny, editors, The Major Transitions in Evolution Revisited, pages 127-140. The MIT Press, Cambridge, MA, 2011.

23. B Calcott and K. Sterelny. Introduction: a dynamic view of evolution. In The Major Transitions in Evolution Revisited, pages 1-14. 2011.

24. A. F. G. Bourke. Principles of Social Evolution. Oxford University Press, Oxford, 2011.

25. Stuart A. West, Roberta M. Fisher, Andy Gardner, and E. Toby Kiers. Major evolutionary transitions in individuality. Proceedings of the National Academy of Sciences, 112(33):10112-10119, 2015.

26. D. W. McShea and C. Simpson. The miscellaneous transitions in evolution. In The Major Transitions in Evolution Revisited, pages 19-33. 2011.

27. Maureen A O'Malley and Russell Powell. Major problems in evolutionary transitions: how a metabolic perspective can enrich our understanding of macroevolution. Biology \& Philosophy, 31(2):159-189, 2016.

28. Daniel H. Janzen. What are dandelions and aphids? The American Naturalist, 111(979):586-589, January 1977.

29. R E Michod, A. M. Nedelcu, and D Roze. Cooperation and conflict in the evolution of individuality. IV. Conflict mediation and evolvability in Volvox carteri. BioSystems, 69(2-3):95-114, May 2003.

30. Henri J. Folse and Joan Roughgarden. What is an individual organism? A multilevel selection perspective. Quarterly Review of Biology, 85(4):447-472, December 2010.

31. Joan E Strassmann and David C Queller. The social organism: congresses, parties, and committees. Evolution, 64(3):605-616, March 2010.

32. Matthijs van Veelen, Julián García, Maurice W Sabelis, and Martijn Egas. Group selection and inclusive fitness are not equivalent; the Price equation vs. models and statistics. Journal of Theoretical Biology, August 2011.

33. Ellen Clarke. The problem of biological individuality. Biological Theory, 5(4):312325, December 2011.

34. J Maynard Smith. Evolutionary progress and levels of selection. In M. H. Nitecki, editor, Evolutionary Progress, pages 219-230. The University of Chicago Press, Chicago, 1988.

35. Dániel Czégel, István Zachar, and Eörs Szathmáry. Multilevel selection as Bayesian inference, major transitions in individuality as structure learning. Royal Society Open Science, 6:190202-190202, 2019.

36. C. R. Darwin. Narrative of the surveying voyages of His Majesty's Ships Adventure and Beagle between the years 1826 and 1836, describing their examination of the southern shores of South America, and the Beagle's circumnavigation of the globe. Journal and remarks. 1832-1836. Henry Colburn, London, 1839.

37. Eva Jablonka and Marion J. Lamb. The evolution of information in the major transitions. Journal of Theoretical Biology, 239(2):236-246, 2006. 
38. Patrick J Keeling. The endosymbiotic origin, diversification and fate of plastids. Philosophical Transactions of the Royal Society of London. Series B, Biological sciences, 365(1541):729-748, March 2010.

39. D. H. Erwin. A public goods approach to major evolutionary innovations. Geobiology, 13(4):308-315, 2015.

40. Austin Booth. Populations and individuals in heterokaryotic fungi: a multilevel perspective. Philosophy of Science, 81(4):612-632, October 2014.

41. R. C. Lewontin. The units of selection. Annual Review of Ecology and Systematics, 1(1):1-18, November 1970.

42. David L. Hull. Units of evolution: a metaphysical essay. In V.J. Jensen and R. Harre, editors, The Philosophy of Evolution, pages 23-44. Harvester Press, Brighton, England, 1981.

43. J.W. Pepper and M.D. Herron. Does biology need an organism concept? Biological Reviews, 83(4):621-627, 2008.

44. M.D. Matthew D. Herron, A. Rashidi, D.E. E Shelton, and W.W. Driscoll. Cellular differentiation and individuality in the "minor" multicellular taxa. Biological Reviews of the Cambridge Philosophical Society, 88(4):844-861, 2013.

45. Tracey Chapman, Göran Arnqvist, Jenny Bangham, and Locke Rowe. Sexual conflict. Trends in Ecology 83 Evolution, 18(1):41-47, January 2003.

46. C. T. Regan. Dwarfed males parasitic on the females in oceanic angler-fishes (Pediculati ceratioidea). Proceedings of the Royal Society B, 97:386-400, 1925.

47. T. W. Pietsch. Dimorphism, parasitism, and sex revisited: modes of reproduction among deep-sea ceratioid anglerfishes (Teleostei: Lophiiformes). Ichthyological Research, 52:207-236, 2005.

48. Eiichiro Kokubo and Shigeru Ida. Formation of protoplanet systems and diversity of planetary systems. The Astrophysical Journal, 581(1):666-680, 2002.

49. Patryk Sofia Lykawka and Tadashi Mukai. Dynamical classification of transneptunian objects: Probing their origin, evolution, and interrelation. Icarus, 189(1):213-232, July 2007.

50. S. Ida and D. N. C. Lin. Toward a deterministic model of planetary formation. III. Mass distribution of short-period planets around stars of various masses. The Astrophysical Journal, 626:1045-1060, 2005.

51. Richard K. Grosberg and Richard R. Strathmann. The evolution of multicellularity: a minor major transition? Annual Review of Ecology, Evolution, and Systematics, 38(1):621-654, 2007.

52. Gregory S. Gavelis and Gillian H. Gile. How did cyanobacteria first embark on the path to becoming plastids?: lessons from protist symbioses. FEMS Microbiology Letters, 365(19), October 2018.

53. Filip Husnik and Patrick J Keeling. The fate of obligate endosymbionts: reduction, integration, or extinction. Current Opinion in Genetics 83 Development, 58-59:1-8, October 2019. 
54. Carol D. von Dohlen, Shawn Kohler, Skylar T. Alsop, and William R. McManus. Mealybug \beta-proteobacterial endosymbionts contain \gamma-proteobacterial symbionts. Nature, 412(6845):433-436, July 2001.

55. Kaoru Kawafune, Yuichi Hongoh, and Hisayoshi Nozaki. A rickettsial endosymbiont inhabiting the cytoplasm of Volvox carteri (Volvocales, Chlorophyceae). Phycologia, 53(1):95-99, January 2014.

56. T Nakada, H. Nozaki, and Masaru Tomita. Another origin of coloniality in volvocaleans: the phylogenetic position of Pyrobotrys arnoldi (Spondylomoraceae, Volvocales). The Journal of Eukaryotic Microbiology, 57(4):379-382, 2010.

57. Stephen R. Fairclough, Mark J. Dayel, and Nicole King. Multicellular development in a choanoflagellate. Current Biology, 20(20):R875-R876, October 2010.

58. Russell Powell and Maureen A. O'Malley. Metabolic and microbial perspectives on the "evolution of evolution". Journal of Experimental Zoology Part B: Molecular and Developmental Evolution, 0(0), 2019.

59. Daniel W. McShea. The hierarchical structure of organisms: a scale and documentation of a trend in the maximum. Paleobiology, 27(2):405-423, 2001.

60. J. Arvid Ågren. Evolutionary transitions in individuality: insights from transposable elements. Trends in Ecology \& Evolution, 29(2):90-96, February 2014.

61. Yohay Carmel and Ayelet Shavit. Operationalizing evolutionary transitions in individuality. Proceedings of the Royal Society B: Biological Sciences, 287(1920):20192805, February 2020. 